\title{
Aberrant Expression of Long Non-Coding RNAs in Newly Diagnosed Type 2 Diabetes Indicates Potential Roles in Chronic Inflammation and Insulin Resistance
}

\author{
Xiaoli Wang ${ }^{\mathrm{a}}$ Xiangyun Chang ${ }^{\mathrm{a}}$ Peipei Zhang ${ }^{\mathrm{a}} \quad$ Ling Fan $^{\mathrm{a}}$ Ting Zhou ${ }^{\mathrm{b}}$ Kan Sun ${ }^{\mathrm{a}}$ \\ aDepartments of Endocrinology and Metabolism, bMedical Experimental Laboratory, First Affiliated \\ Hospital, School of Medicine, Shihezi University, Shihezi, Xinjiang, China
}

\section{Key Words}

Type 2 diabetes • IncRNA • Inflammation • Insulin resistance

\begin{abstract}
Background/Aims: Long non-coding RNAs (IncRNAs) have emerged as key players in several biological processes and complex diseases. The risk of type 2 diabetes (T2D) is determined by a combination of environmental factors and genetic susceptibility. The purpose of this study was to identify aberrant IncRNAs involved in T2D pathogenesis. Methods: Microarray analysis was performed using whole blood samples from patients newly diagnosed with T2D and healthy controls. Pathway and Gene Ontology (GO) analyses were utilized to annotate the target genes. Coding non-coding co-expression (CNC) analysis was performed to construct a co-expression network. Results: We found 55 IncRNAs and 202 mRNAs were differentially expressed in the T2D group compared to the healthy control group. Pathway and GO analyses demonstrated that dysregulated mRNAs were mainly associated with immune regulation, inflammation, and insulin resistance, whereas CNC analysis identified 10 pairs of co-expressed IncRNA-mRNAs in our patient cohort $(R>0.99)$. Furthermore, expression of the top three upregulated IncRNAs in the T2D group was correlated with measures of glycometabolism $(P$ $<0.05)$. Conclusion: This study identified aberrantly expressed IncRNAs and mRNAs in Han Chinese patients with T2D, and demonstrated that dysregulated IncRNAs may have roles in T2D pathogenesis through regulation of inflammation and insulin resistance.
\end{abstract}

(C) 2017 The Author(s)

Published by S. Karger AG, Basel

\section{Introduction}

Diabetes mellitus (DM) is one of the most common chronic diseases in the world characterized by elevated plasma glucose levels, and type 2 diabetes (T2D) accounts for most patients with DM. According to the World Health Organization (WHO), in 2015 there

X. Wang and X. Chang contributed equally to this work. 


\section{Cellular Physiology Cell Physiol Biochem 2017;43:2367-2378

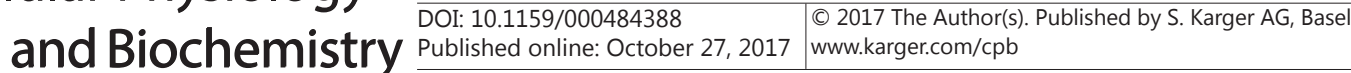 \\ Wang et al.: Long Non-Coding RNAs and Type 2 Diabetes in Han Chinese}

were 415 million patients with T2D globally, which is predicted to rise to 642 million by 2040 [1]. The risk of acquiring T2D is determined by a combination of environmental factors and genetic susceptibility.

Long non-coding RNAs (lncRNAs) are defined as non-encoding RNA transcripts longer than 200 bp [2]. LncRNAs were previously considered as "transcriptional noise" because of their poor conservation among species and low expression. To date, over 7000 lncRNAs have been identified in the human genome, of which only a small number have been ascribed a function [3]. It has been realized that lncRNAs regulate multiple biological processes [4], with an increasing number of studies demonstrating that lncRNAs play important roles in cell differentiation, tissue development, chromosome inactivation, and epigenetic modification [5-7]. Furthermore, the link between genetically controlled lncRNA expression and human disease has been established [8], with growing evidence of their involvement in glucose homeostasis and DM [9-12]. Over 1000 lncRNAs that are relevant to $\beta$ cell programming and diabetes pathophysiology have been identified in pancreatic beta cells [13].

A core mechanism of T2D is insulin resistance (IR), which is a condition in that targeted cells fail to respond to insulin. IR is characterized by a decrease in insulin signaling [14]. Although it is widely accepted that low-grade chronic inflammation is an important factor in the pathogenesis of IR $[15,16]$, the molecules and mechanisms that regulate inflammation and the IR process in T2D are not fully understood. However, non-coding RNAs were implicated in the regulation of these processes [17-20].

The purpose of this study is to identify lncRNAs associated with T2D by examining lncRNA and mRNA expression profiles from whole blood samples of patients newly diagnosed with T2D compared to healthy individuals. We also investigated whether there were correlations between the expression level of aberrantly expressed lncRNAs and different measures of metabolic processes. We demonstrate that dysregulated lncRNAs may have roles in the pathogenesis of T2D through regulation of inflammation and IR.

\section{Materials and Methods}

Ethics

This study was conducted in accordance with the Declaration of Helsinki and performed with the approval from the Ethics Committee of the First Affiliated Hospital, School of Medicine Shihezi University (China). Informed consent was provided by all participants before entering the study.

\section{Subjects}

We enrolled individuals $\geq 35$ years old at the time of their visit at the First Affiliated Hospital in Shihezi (China) from October 2015 to December 2016. The diagnosis of T2D was based on the 1999 WHO diagnostic criteria [21]. Our control group consisted of healthy volunteers without DM who were recruited from communities in Shihezi in China. Demographic and clinicopathological information, such as age, ethnicity, previous diseases, medication, smoking, and drinking habits, was obtained via a questionnaire. All participants were Han Chinese and had not been treated with any hypoglycemic agents. Individuals with a history of cancer, pregnancy, lactation, immunodeficiency, chronic organ disease, or infectious disease or patients who were receiving immunosuppressive or hormone-containing drugs were excluded.

The initial 'microarray cohort' (discovery cohort) was composed of six patients newly diagnosed with T2D and six healthy volunteers, whereas the validation cohorts were composed of 60 newly diagnosed patients with T2D and 60 healthy control individuals.

\section{Anthropometric and biochemical measurements}

For all participants, anthropometric and body composition measurements were performed before breakfast. Body mass index (BMI) was calculated as weight (kg) divided by the square of the height $(\mathrm{m})$. A standard $75 \mathrm{~g}$ oral glucose tolerance test was conducted for all subjects after overnight fasting (longer than $10 \mathrm{~h}$ ). In brief, $75 \mathrm{~g}$ of glucose was ingested within $5 \mathrm{~min}$ and peripheral blood samples were collected in the fasting state and $2 \mathrm{~h}$ after glucose ingestion. Blood samples were withdrawn from the cubital vein 


\section{Cellular Physiology Cell Physiol Biochem 2017;43:2367-2378

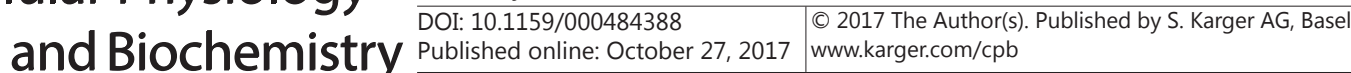 \\ Wang et al.: Long Non-Coding RNAs and Type 2 Diabetes in Han Chinese}

and collected in evacuated sample tubes containing preservatives (heparin sodium). The tubes were stored at $4{ }^{\circ} \mathrm{C}$ until centrifugation. In addition, $1 \mathrm{~mL}$ fresh whole blood samples collected in evacuated sample tubes containing ethylene diamine tetraacetic acid were collected for measurement of hemoglobin A1c (HbA1c) level using an automated D10 analyzer (Bio-Rad, Hercules, CA, USA). Fasting plasma glucose (FPG), 2-hour plasma glucose (2hPG), total cholesterol (TC), triglyceride (TG), high-density lipoprotein cholesterol (HDL-C), and low-density lipoprotein cholesterol (LDL-C) levels were analyzed enzymatically using an autoanalyzer (Hitachi, Tokyo, Japan). Fasting and 2-hour plasma insulin (2hINS) levels were detected using electrochemiluminescence (E170, Roche, Basel, Switzerland).

\section{Total RNA extraction and purification}

Total RNA was extracted from peripheral blood using the PAXgene Blood RNA kit (Qiagen, Dusseldorf, Germany) for isolation and purification according to the manufacturer's instructions and as described previously [22]. Briefly, approximately $2.5 \mathrm{~mL}$ of blood from each subject was incubated for $2 \mathrm{~h}$ at room temperature in PAXgene ${ }^{\circledR}$ Blood RNA tubes (Becton Dickinson, Franklin Lakes, NJ, USA) followed by centrifugation for $10 \mathrm{~min}$ at $3000 \times \mathrm{g}$. Cell pellets were then incubated with a mixture of $300 \mu \mathrm{L}$ binding buffer and $40 \mu \mathrm{L}$ of proteinase $\mathrm{K}$ for $10 \mathrm{~min}$ at $55^{\circ} \mathrm{C}$ with shaking. The resultant lysate was centrifuged for 3 min in a PAXgene ${ }^{\circledR}$ Shredder spin column at $16300 \times \mathrm{g}$, and the supernatant was then mixed with $350 \mu \mathrm{L}$ of $100 \%$ ethanol in the PAXgene $\AA$ RNA spin column followed by centrifugation for 1 min at $16300 \times \mathrm{g}$. The purified RNA was eluted by elution buffer, denatured by incubating at $65^{\circ} \mathrm{C}$ for $5 \mathrm{~min}$, chilled on iced, and stored at $-20{ }^{\circ} \mathrm{C}$ until use in microarray and gene expression studies.

\section{RNA labeling and hybridization}

Double-stranded complementary DNA (cDNA) was synthesized from total RNA according to the manufacturer's instructions and labeled with a fluorescent dye. Labeled cDNA was denatured at $95{ }^{\circ} \mathrm{C}$ for 3 min in hybridization solution and hybridized for $16 \mathrm{~h}$ at $45^{\circ} \mathrm{C}$ on GeneChip Human Transcriptome Array 2.0 microarrays (Affymetrix, Santa Clara, CA, USA), which covers $>245000$ protein-coding transcripts and $>40$ 000 non-coding transcripts. Following hybridization, slides were washed and dried prior to data collection using Microarray Scanner 3000 (Affymetrix, Santa Clara, CA, USA).

\section{Microarray analysis}

Transcriptome Analysis Console software (Affymetrix) was used to analyze the expression profiles of IncRNAs and mRNAs. Differentially expressed genes were identified based on threshold changes of $\geq 2$-fold or $\leq-2$-fold and $P$ values $<0.05$. The data were normalized and hierarchically clustered with CLUSTER 3.0. Data were performed to be Tree Visualization with Java Treeview software (Stanford University School of Medicine, Stanford, CA, USA)

\section{Pathway and Gene Ontology (GO) analysis}

Pathway analysis was a functional analysis for mapping genes to pathways based on Kyoto Encyclopedia of Genes and Genomes (KEGG). The enriched genes in the KEGG pathway were statistically calculated by hypergeometric distribution. Gene Ontology (GO) was also used to explore the potential molecular function of the differentially expressed mRNAs (http://geneontology.org/).

\section{Construction of the coding non-coding gene co-expression network}

The coding non-coding co-expression (CNC) network was constructed based on correlation analysis between the differentially expressed IncRNAs and mRNAs. The network was drawn using the open source bioinformatics software Cytoscape (http://www.cytoscape.org).

\section{Quantitative real-time PCR and statistical analysis}

Primers for quantitative real-time PCR (qRT-PCR) were designed based on the lncRNA sequences from NONCODE (http://www.noncode.org), and were synthesized and purified at Shenggong (Shanghai, China). The reverse transcription reactions on extracted total RNA were performed using a cDNA synthesis kit (Qiagen, Dusseldorf, Germany) and a 7500 Fast RT-PCR System (Applied Biosystems). qRT-PCR was performed using the ABI 7500 Fast. The qRT-PCR cycle was $95^{\circ} \mathrm{C}$ for $2 \mathrm{~min}$, followed by 40 cycles of $95{ }^{\circ} \mathrm{C}$ for $5 \mathrm{~s}$ and $60^{\circ} \mathrm{C}$ for $30 \mathrm{~s}$, and a final melting curve analysis from $60-95^{\circ} \mathrm{C}$. qRT-PCR results were quantified 


\section{Cellular Physiology Cell Physiol Biochem 2017;43:2367-2378

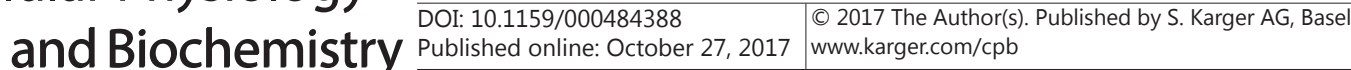 \\ Wang et al.: Long Non-Coding RNAs and Type 2 Diabetes in Han Chinese}

using the $2^{-\triangle \mathrm{ct}}$ method against $\beta$-actin (ACTB) for normalization. The data represent the means of three experiments. The primers used in qRT-PCR were listed in Table 1. Because data of qRT-PCR were abnormally distributed, the expression level of IncRNAs between healthy control and T2D group were analyzed using Mann - Whitney $U$ test. Spearman's correlation analyses were employed to verify the relationship between expression level of lncRNAs and metabolic features. $P<0.05$ was considered statistically significant.

\section{Results}

IncRNA expression profiles in patients with T2D

The microarray cohort was composed of six patients newly diagnosed with T2D and six healthy volunteers. Their clinical and demographic characteristics were shown (for all online suppl. material, see www. karger.com/doi/10.1159/000484388) in Table S1. From our microarray analysis, we detected 13921 IncRNAs, of which 55 IncRNAs were differently expressed in the T2D group compared to the healthy control group (Fig. 1a). Of these 55 lncRNAs identified in our patient cohort, we found that 39 lncRNAs were upregulated and 16 lncRNAs were downregulated (see online suppl. material, Tables S2 and S3).
Table 1. Primers for qRT-PCR detection of lncRNAs

\begin{tabular}{lc}
\hline Target & \multicolumn{1}{c}{ Primer } \\
\hline n342533 & Forward, 5'-TGGCTGTTCTGAAGAGTGTTACTG-3' \\
& Reverse, 5'-GCCAGAGGGTCTGTAGTTCTGAT-3' \\
n341578 & Forward, 5'-GCTAAATCAGAGAGGCAGGAAAG-3' \\
& Reverse, 5'-ATGCATCAATATCCTCCCAATTA-3' \\
n341270 & Forward, 5'-AGGCTGCTGGACTGGAACTC-3' \\
& Reverse, 5'-ATACACTGGGTGGGCTCCTT-3' \\
n336302 & Forward, 5'-CAATAATCACGGCTGCCAAG-3'
\end{tabular}

Reverse, 5'-GGCCTTTCCAGGTTGGAACT-3'

n405950 Forward, 5'-GCGGTACAGTCTGGGCATTC-3'

Reverse, 5'-AGATCAGGGAGGGCTGCATT-3'

n341520 Forward, 5'-GGAGGCAAACGCTGTGTTATC-3'

Reverse, 5'-AGGAGAAGCAGCAGCATCAGA-3'

n338909 Forward, 5'-TCCCACCCACTCAGCTCACT-3'

Reverse, 5'-ACCAGGAAGCTCACGGACAC-3'

n325833 Forward, 5'-TGTGGAGTGGGTAGCCTCCA-3'

Reverse, 5'-CCGAGCTTTAGGTAATTCTCACAG-3'

n410159 Forward, 5'-ATTGCTGTGGCGTCCTGC-3'

Reverse, 5'-TCGATTACCCCCATCAGGC-3'

n342476 Forward, 5'-CTCTGCCTCGCAGGTTCAAG-3'

Reverse, 5'-GGTGGATCACCTGAGGTCAAG-3'

ACTB Forward, 5'-ACTGGGACGACATGGAGAAA-3'

Reverse, 5'-TAGCACAGCCTGGATAGCAA-3'

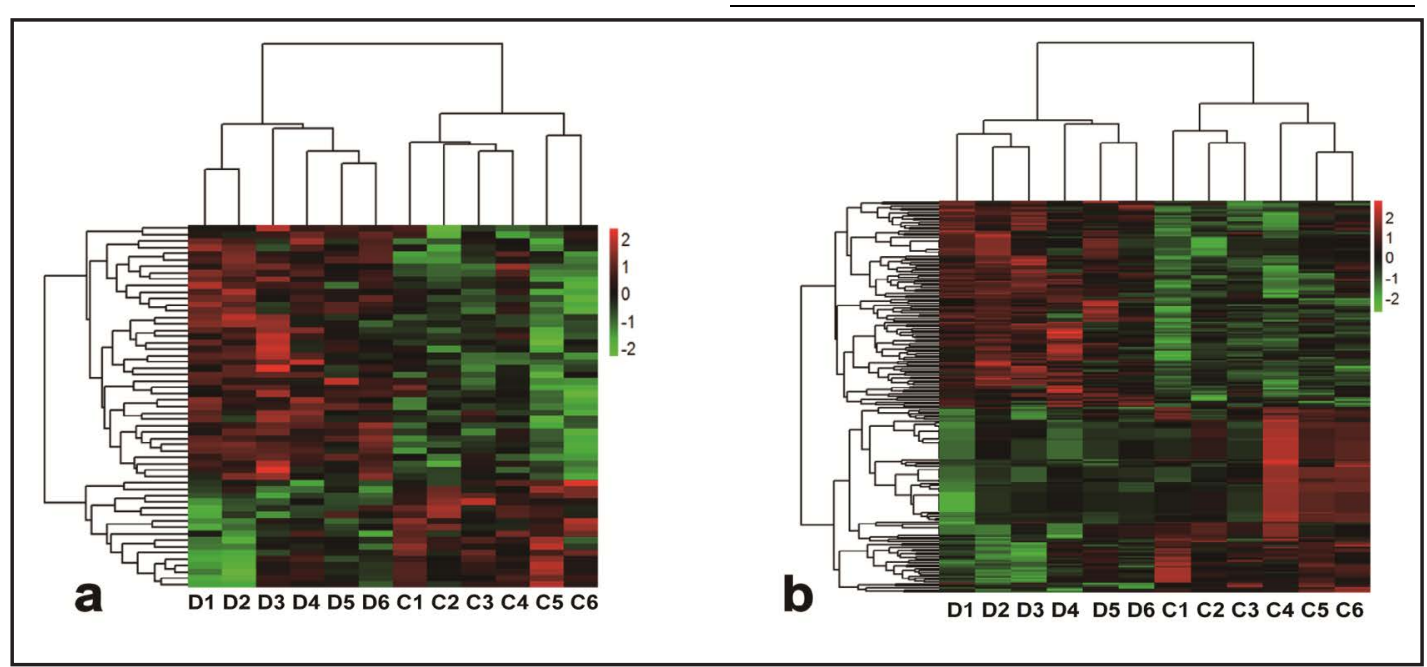

Fig. 1. Differential expression of long non-coding RNAs (lncRNAs) and mRNAs in patients with type 2 diabetes (T2D) and controls. Hierarchical clustering analysis of (a) 55 lncRNAs and (b) 202 mRNAs that were differentially expressed in the two groups of participants. Expression values are represented as red and green, which indicate upregulation and downregulation respectively, in patients (D1, D2, D3, D4, D5, and D6) compared to that found in control individuals (C1, C2, C3, C4, C5, and C6). 


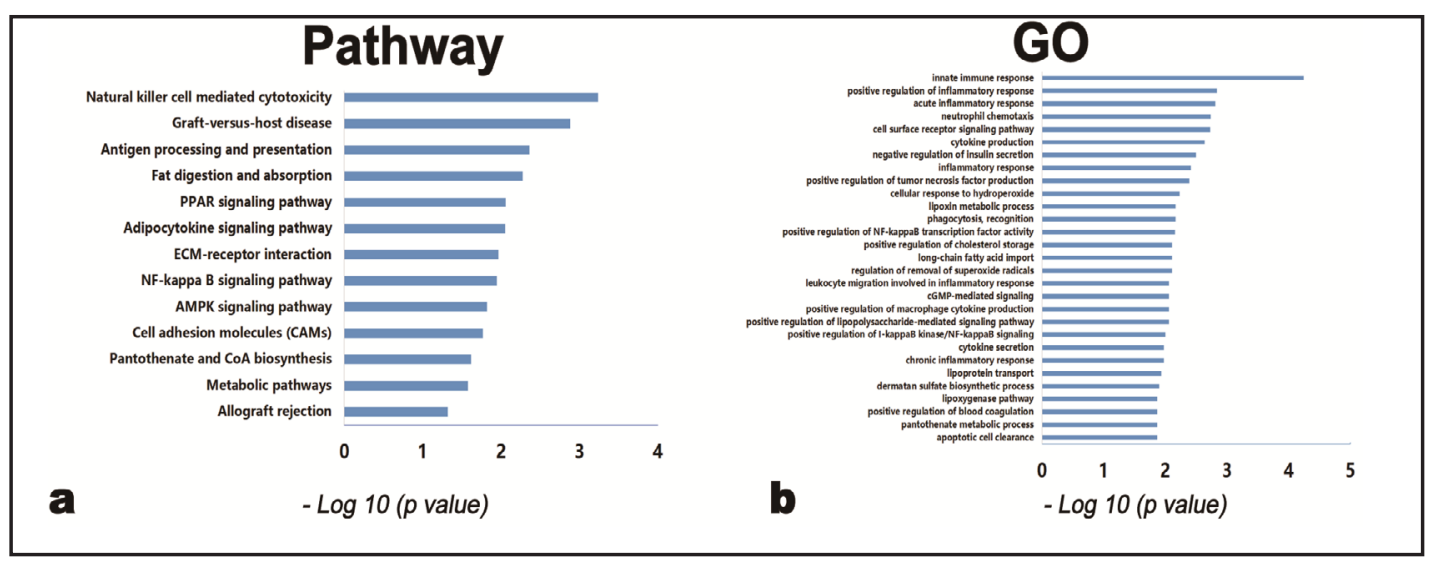

Fig. 2. The pathways (a) or Gene Ontology (GO) (b) terms identified with the aberrantly expressed mRNAs found in the patient cohort compared to the control cohort.

Table 2. Correlation between IncRNAs and mRNAs (only correlation $>0.99$ genes are shown). ${ }^{\text {a }}$ Probe set

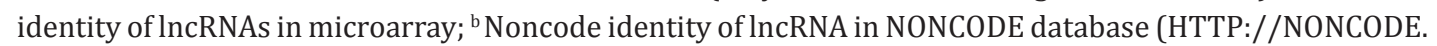
org); ${ }^{\text {P }}$ robe set identity of mRNAs in microarray

\begin{tabular}{|c|c|c|c|c|c|c|}
\hline LncRNA (Probe set ID) a & Noncode ID b & Correlation & P value & mRNA (Probe set ID) $^{c}$ & Gene ID & Gene symbol \\
\hline TC19002237.hg.1 & n336109 & 0.995690 & 0.001282 & TC06002121.hg.1 & 8875 & VNN2 \\
\hline TC04002272.hg.1 & n379706 & 0.995335 & 0.032954 & TC06000051.hg.1 & 9450 & LY86 \\
\hline TC21000719.hg.1 & n384014 & 0.995106 & 0.002139 & TC21000167.hg.1 & 3772 & KCNJ15 \\
\hline TC13000982.hg.1 & n382000 & 0.994872 & 0.018456 & TC01003260.hg.1 & 6283 & S100A12 \\
\hline TC06003855.hg.1 & n409772 & 0.994590 & 0.001282 & TC06002121.hg.1 & 8875 & VNN2 \\
\hline TC03002627.hg.1 & n382000 & 0.994056 & 0.004603 & TC07000509.hg.1 & 948 & CD36 \\
\hline TC19002237.hg.1 & n336109 & 0.993600 & 0.001156 & TC0X000052.hg.1 & 51311 & TLR8 \\
\hline TC0X001624.hg.1 & n408024 & 0.991440 & 0.012212 & TC01003261.hg.1 & 6279 & S100A8 \\
\hline TC08002477.hg.1 & n341587 & 0.991300 & 0.004603 & TC07000509.hg.1 & 948 & CD36 \\
\hline TC19002237.hg.1 & n336109 & 0.990320 & 0.000051 & TC21000167.hg.1 & 3772 & KCNJ15 \\
\hline
\end{tabular}

mRNA expression profiles in T2D

We also identified 44699 coding RNAs from microarray analysis. Of the 202 mRNAs that have significantly different expression between the T2D group and the healthy control group (Fig. 1b), 106 mRNAs were upregulated (see online suppl. material, Table S4) and 96 mRNAs were downregulated (see online suppl. material, Table S5).

\section{Pathway and GO analyses}

We found using pathway analysis that the differentially expressed mRNAs identified in our Han Chinese cohort with T2D were involved in 13 pathways, including natural killer cellmediated cytotoxicity, fat digestion and absorption, the nuclear peroxisome proliferatoractivated receptor (PPAR) signaling pathway, the adipocytokine signaling pathway, the NF-kappa B signaling pathway, et al. (Fig. 2a). Using GO biological process analysis, we also found that the dysregulated mRNAs were involved in 29 items, including innate immune response, positive regulation of the inflammation response, neutrophil chemotaxis, cytokine production, negative regulation of insulin secretion, et al. (Fig. 2b). 


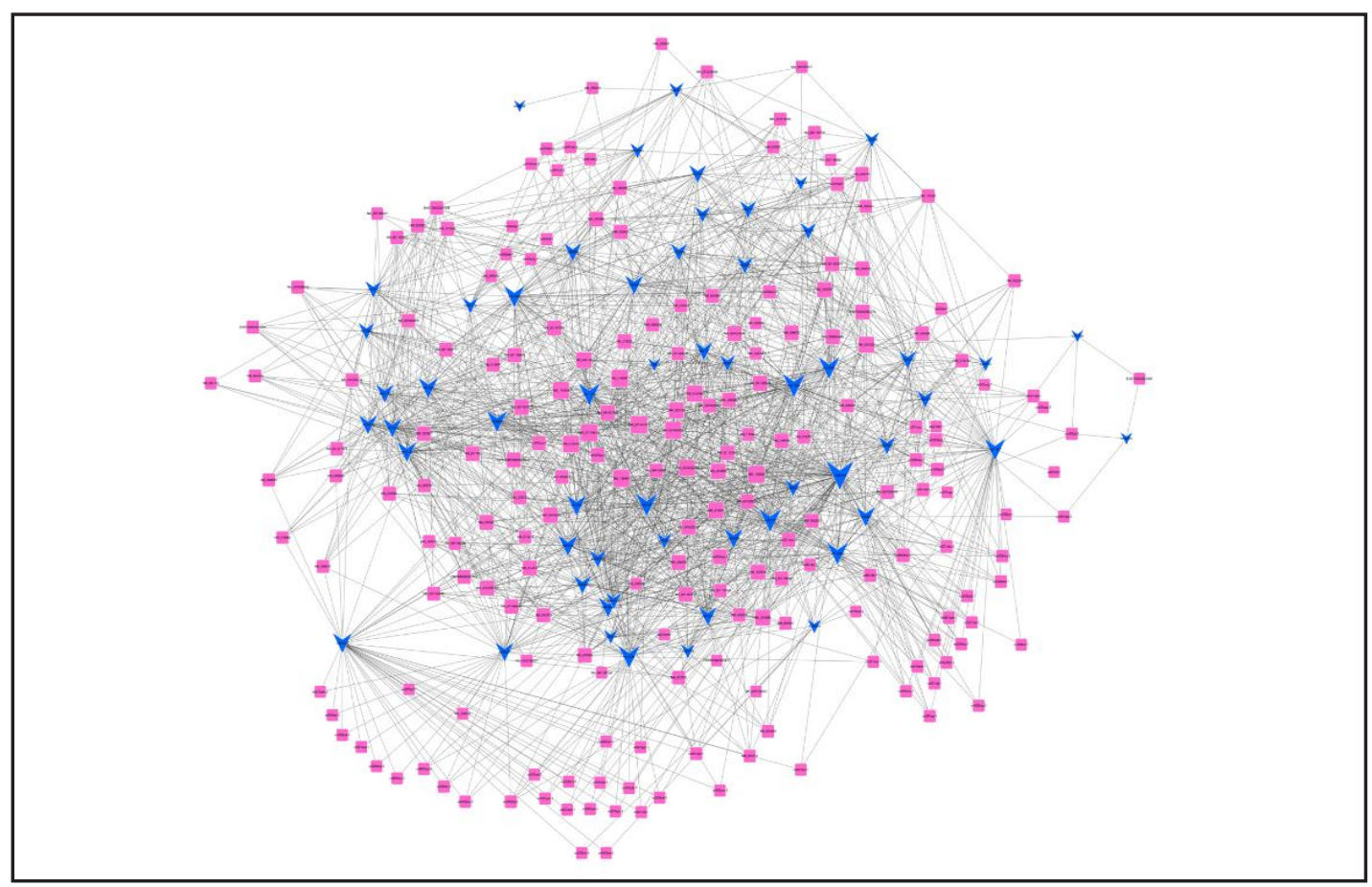

Fig. 3. The coding non-coding co-expression network of differentially expressed long non-coding RNAs and mRNAs found in the patient cohort compared to the control cohort. Red box represents mRNA, blue triangle represents IncRNA.

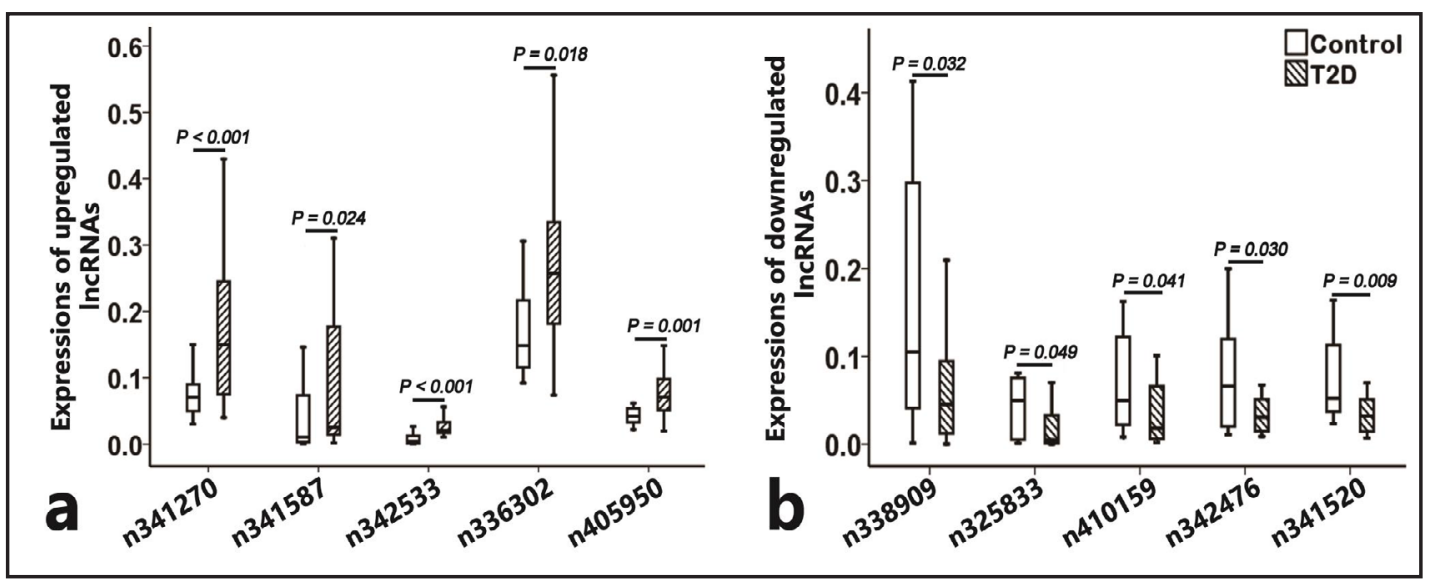

Fig. 4. Validation of differentially expressed long non-coding RNAs (lncRNAs) in microarrays by quantitative PCR (qPCR). (a) Five upregulated and (b) five downregulated lncRNAs from microarray data were assayed by qPCR using $\beta$-actin (ACTB) as a housekeeping gene for normalization.

\section{CNC network of IncRNAs and mRNAs}

There were 55 lncRNAs and 202 mRNAs for CNC network analysis, which derived 256 network nodes and 2070 co-expression pairs of IncRNAs and mRNAs (Fig. 3). Our analysis of the CNC network indicated that a single lncRNA is correlated with one to tens of mRNAs and vice versa. Ten IncRNA-mRNA pairs have a co-expression relationship $(\mathrm{R}>0.99)$. The target mRNAs were lymphocyte antigen 86 (LY86), S100 calcium binding protein A12 (S100A12), S100 calcium binding protein A8 (S100A8), CD36, toll-like receptor 8 (TLR8), Vanin-2 (VNN2), and potassium inwardly-rectifying channel, subfamily J, member 15 (KCNJ15).

\section{KARGER}


Table 2 lists the differentially expressed mRNAs and their co-expressed lncRNAs in the CNC network with a correlation coefficient $>0.99$ and $P<0.05$.

\section{qRT-PCR validation}

To independently validate the identified dysregulated lncRNAs, five upregulated and five downregulated lncRNAs were randomly chosen from differentially expressed lncRNAs (fold changes $\geq 2.0$ or $\geq-2.0$ ). qRT-PCR was performed to compare the expression levels of these differentially expressed lncRNAs in the T2D group $(n=60)$ versus those found in the healthy control group $(n=60)$. As shown in Fig. 4, our qRT-PCR results are consistent with the findings from our microarray data.

Correlation of IncRNA expression with metabolic features

To investigate whether there is a correlation between differentially regulated lncRNAs in our cohort of T2D and metabolic features, expression of the top three upregulated IncRNAs (n342533, n335556, and n336109) were examined by qPCR in 60 pairs of whole blood RNA samples. The clinical features of the T2D group and the healthy control group are shown in Table 3 . In addition to confirming that expression of n342533, n335556, and n336109 was significantly increased in the T2D group compared to the healthy control group (all $P<0.05$ ) (Fig. 5), we found using correlation analysis that n342533 was positively correlated with BMI, FPG, 2hPG, HbA1c, homeostatic model assessment (HOMA)IR and ALT (all $P<0.05$ ) and negatively correlated with HDL-C and HOMA- $\beta$ (both $P$ $<0.05$ ). We also found that n335556 was positively correlated with FPG, HbA1c, and HOMA-IR (all $P<0.05$ ) and negatively correlated with 2hINS $(P<0.05)$, whereas n336109 was positively correlated with FPG, 2hPG, HbA1c, and HOMA-IR (all $P$ $<0.05$ ) and negatively correlated with HDL-C, 2hINS, and
Table 3. Demographic and metabolic characteristics of the T2D group and healthy control group. Data are reported as means \pm standard deviation, number (\%) or median (interquartile range). BMI, body mass index; FPG, fasting plasma glucose; 2hPG, 2-hour plasma glucose; HbA1c, haemoglobin A1c; FINS, fasting insulin; 2hINS, 2-hour insulin; HOMA-IR, homeostatic model assessment of insulin resistance; HOMA- $\beta$, homeostatic model assessment of $\beta$ cell function; TG, Triglyceride; TC, total cholesterol; HDL-C, high density lipoprotein cholesterol; LDL-C, low density lipoprotein cholesterol; ALT, alanine transaminase; AST, aspartase aminotransferase

\begin{tabular}{|c|c|c|c|}
\hline Variable & $\begin{array}{l}\text { T2D } \\
(n=60)\end{array}$ & $\begin{array}{l}\text { Healthy control } \\
(n=60)\end{array}$ & $P$ value \\
\hline Male (\%) & 61.7 & 58.3 & 0.426 \\
\hline Age (year) & $50.4 \pm 13.4$ & $51.0 \pm 9.0$ & 0.792 \\
\hline Current smokers (\%) & 21.7 & 20.0 & 0.798 \\
\hline Current drinkers (\%) & 20.0 & 21.7 & 0.500 \\
\hline Systolic blood pressure (mmHg) & $129 \pm 13$ & $127 \pm 14$ & 0.366 \\
\hline Diastolic blood pressure (mmHg) & $77 \pm 11$ & $77 \pm 10$ & 0.871 \\
\hline $\operatorname{BMI}\left(\mathrm{kg} / \mathrm{m}^{2}\right)$ & $26.3 \pm 3.0$ & $25.4 \pm 2.7$ & 0.086 \\
\hline $\mathrm{FPG}(\mathrm{mmol} / \mathrm{L})$ & $9.56 \pm 3.13$ & $4.93 \pm 0.47$ & $<0.001$ \\
\hline 2hPG (mmol/L) & $17.61 \pm 4.31$ & $6.40 \pm 0.80$ & $<0.001$ \\
\hline $\operatorname{HbA} 1 \mathrm{c}(\%)$ & $9.1 \pm 2.1$ & $5.5 \pm 0.4$ & $<0.001$ \\
\hline FINS $(\mu \mathrm{U} / \mathrm{mL})$ & $58.42 \pm 40.53$ & $57.82 \pm 16.50$ & 0.916 \\
\hline $2 \mathrm{hINS}(\mu \mathrm{U} / \mathrm{mL})$ & $193.15 \pm 117.76$ & $288.44 \pm 113.66$ & $<0.001$ \\
\hline HOMA - IR & $3.17(1.72-4.72)$ & $1.58(1.31-2.19)$ & $<0.001$ \\
\hline HOMA - $\beta$ & $31.84 \pm 19.93$ & $124.77 \pm 61.64$ & $<0.001$ \\
\hline $\mathrm{TG}(\mathrm{mmol} / \mathrm{L})$ & $2.21 \pm 1.28$ & $1.43 \pm 0.75$ & $<0.001$ \\
\hline $\mathrm{TC}(\mathrm{mmol} / \mathrm{L})$ & $4.67 \pm 0.88$ & $4.40 \pm 0.89$ & 0.105 \\
\hline HDL - C (mmol/L) & $0.96 \pm 0.25$ & $1.27 \pm 0.35$ & $<0.001$ \\
\hline LDL - C (mmol/L) & $2.83 \pm 0.88$ & $2.71 \pm 0.78$ & 0.434 \\
\hline $\operatorname{ALT}(\mathrm{U} / \mathrm{L})$ & $25.8 \pm 11.1$ & $21.2 \pm 14.6$ & 0.047 \\
\hline AST(U/L) & $22.8 \pm 10.9$ & $21.9 \pm 8.9$ & 0.608 \\
\hline Urea (mmol/L) & $5.54 \pm 1.71$ & $5.11 \pm 1.43$ & 0.144 \\
\hline Creatinine $(\mu \mathrm{mol} / \mathrm{L})$ & $65.44 \pm 17.06$ & $64.21 \pm 20.59$ & 0.732 \\
\hline
\end{tabular}


Table 4. Correlations between expressions of top 3 upregulated IncRNAs and metabolic characteristics. BMI, body mass index; HDL-C, high density lipoprotein cholesterol; FPG, fasting plasma glucose; 2hPG, 2-hour plasma glucose; HbA1c, haemoglobin A1c; FINS, fasting insulin; 2hINS, 2-hour insulin; HOMA-IR, homeostatic model assessment of insulin resistance; HOMA- $\beta$, homeostatic model assessment of $\beta$ cell function; ALT, alanine transaminase

\begin{tabular}{|c|c|c|c|c|c|c|}
\hline \multirow{2}{*}{ Demographic and metabolic characteristics } & \multicolumn{2}{|c|}{ n342533 } & \multicolumn{2}{|c|}{ n335556 } & \multicolumn{2}{|c|}{ n336109 } \\
\hline & $\mathrm{r}$ & $P$ value & $\mathrm{r}$ & P value & $\mathrm{r}$ & Pvalue \\
\hline \multicolumn{7}{|l|}{ Model 1} \\
\hline BMI $\left(\mathrm{kg} / \mathrm{m}^{2}\right)$ & 0.288 & 0.023 & 0.187 & 0.146 & 0.198 & 0.123 \\
\hline HDL-C (mmol/L) & -0.313 & 0.014 & -0.156 & 0.229 & -0.309 & 0.016 \\
\hline FPG (mmol/L) & 0.611 & $<0.001$ & 0.360 & 0.004 & 0.674 & $<0.001$ \\
\hline $2 \mathrm{hPG}(\mathrm{mmol} / \mathrm{L})$ & 0.546 & $<0.001$ & 0.217 & 0.090 & 0.604 & $<0.001$ \\
\hline HbA1c (\%) & 0.578 & $<0.001$ & 0.332 & 0.009 & 0.602 & $<0.001$ \\
\hline FINS $(\mu \mathrm{U} / \mathrm{mL})$ & 0.177 & 0.173 & 0.167 & 0.198 & -0.081 & 0.536 \\
\hline $2 \mathrm{hINS}(\mu \mathrm{U} / \mathrm{mL})$ & -0.179 & 0.164 & -0.300 & 0.018 & -0.307 & 0.015 \\
\hline HOMA - IR & 0.323 & 0.010 & 0.441 & $<0.001$ & 0.374 & 0.003 \\
\hline НОМА - $\beta$ & -0.510 & $<0.001$ & -0.227 & 0.075 & -0.649 & $<0.001$ \\
\hline ALT (mmol/L) & 0.335 & 0.008 & 0.036 & 0.784 & 0.223 & 0.084 \\
\hline \multicolumn{7}{|l|}{ Model 2 adjusted age, gender, BMI and HDL - C } \\
\hline FPG (mmol/L) & 0.391 & 0.003 & 0.305 & 0.022 & 0.614 & $<0.001$ \\
\hline 2hPG (mmol/L) & 0.272 & 0.042 & 0.418 & 0.001 & 0.620 & $<0.001$ \\
\hline HbA1c (\%) & 0.420 & 0.001 & 0.357 & 0.007 & 0.584 & $<0.001$ \\
\hline FINS $(\mu \mathrm{U} / \mathrm{mL})$ & 0.385 & 0.003 & 0.255 & 0.058 & 0.062 & 0.650 \\
\hline $2 \mathrm{hINS}(\mu \mathrm{U} / \mathrm{mL})$ & -0.007 & 0.958 & -0.247 & 0.066 & -0.257 & 0.056 \\
\hline HOMA - IR & 0.587 & $<0.001$ & 0.437 & 0.001 & 0.528 & $<0.001$ \\
\hline НОМА - $\beta$ & -0.188 & 0.165 & -0.253 & 0.060 & 0.381 & 0.004 \\
\hline
\end{tabular}

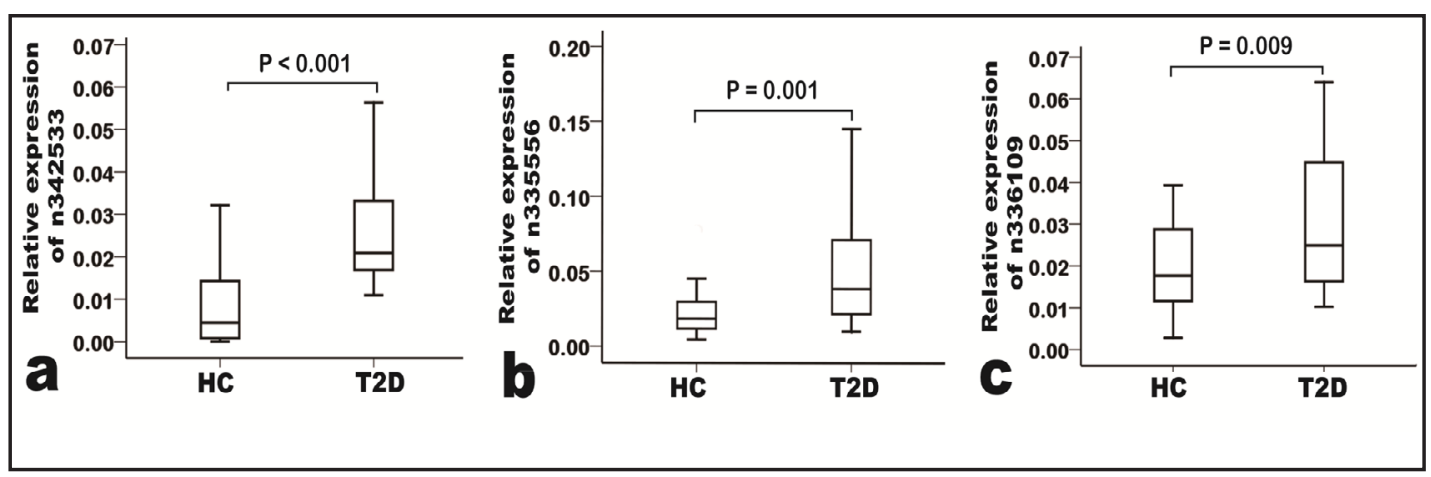

Fig. 5. Expression levels of the long non-coding RNAs (a) n342533, (b) n335556, and (c) n336109 in 60 patients with type 2 diabetes (T2D) compared to those found in 60 healthy controls (HC) by quantitative PCR (qPCR).

HOMA- $\beta$ (all $P<0.05$; Table 4). Following adjustments for age, gender, BMI, and HDL-C, we found that these top three IncRNAs were positively correlated with FPG, 2hPG, HbA1c, and HOMA-IR (all $P<0.05$ ), and n336109 remained negatively correlated with HOMA- $\beta$ (both $P$ $<0.05$; Table 4).

\section{Discussion}

The present study compared IncRNA and mRNA expression profiles in whole blood of six patients newly diagnosed with T2D and six healthy individuals. We identified differentially expressed lncRNAs $(n=55)$ and mRNAs $(n=202)$ in our T2D group compared to our control group, subsets of both were validated by qPCR. We also performed pathway and GO analyses and found that the dysregulated mRNAs associated with T2D were mainly involved in inflammation, immune response, insulin resistance, and the regulation of insulin secretion, whereas using CNC analysis, we found 10 pairs of lncRNAs and mRNAs were co-expressed. 


\section{Cellular Physiology Cell Physiol Biochem 2017;43:2367-2378

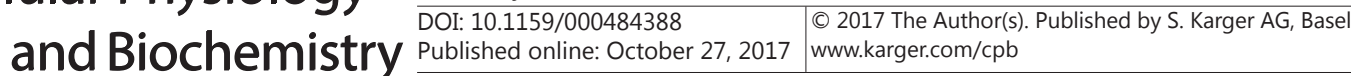 \\ Wang et al.: Long Non-Coding RNAs and Type 2 Diabetes in Han Chinese}

Furthermore, the expression level of the top three upregulated lncRNAs were correlated with variables of glycometabolism and HOMA-IR in our cohort of Han Chinese patients with T2D. To our knowledge, this is the first study to investigate lncRNA expression profile in T2D of Han Chinese population.

T2D is characterized by impaired insulin secretion and IR. The pathogenesis of T2D has been studied for several decades, and although the main cause of the disease is not properly understood, it is widely accepted that interactions between genetic and environmental factors contribute to this widespread condition [23]. The development of IR is contributed by chronic low-grade systemic inflammation [24] that is found in adipose tissue, liver, endocrine pancreas, skeletal muscle, and immune cells [25]. A previous study described lncRNA expression profiles of human pancreatic islets in deceased donors [10]. Although these findings were important towards understanding the involvement of islets and $\beta$-cell dysfunction during the development of T2D, they could not clarify the pathogenesis of IR. Using whole blood samples to examine the expression profiles of IncRNAs and mRNAs in T2D and healthy control groups, the findings from our study contribute towards an improved understand of the pathogenesis of T2D and related conditions, such as immunometabolic disorder, systematic inflammation, and IR.

In the present study, pathway analysis indicated that dysregulated mRNAs were mainly involved in natural killer cell mediated cytotoxicity, antigen processing and presentation, the PPAR signaling pathway, and the adipocytokine signaling pathway in our cohort of Han Chinese patients with T2D. These pathways concerned biological processes of immune regulation, inflammation, or metabolism. Several studies reported a role of the immune system in glucose intolerance and T2D. Defects in invariant natural killer T (INKT) cell populations, which are a subset of natural killer T cells, have been reported in patients with T2D or obesity [26]. Furthermore, INKT cells present in adipose tissue may exert a regulatory role against obesity and associated metabolic disorders [27]. PPARs are fundamentally involved in regulating energy homeostasis. For example, PPAR $\gamma$ is a master regulator of gene expression for metabolism, inflammation, and other pathways in many cell types [28]. Adipose tissue plays a key role in the development of T2D through the secretion of adipokines, such as leptin, adiponectin, and monocyte chemotactic protein-1 [29]. In this study, we found using GO analysis that differentially expressed mRNAs were also most frequently involved in inflammation and immune processes, which coincide with our findings from pathway analysis.

The major function of lncRNAs is to regulate the expression of protein-coding genes [30]. In this study, we identified the biological processes associated with those differentially expressed mRNAs identified in our cohort of T2D. While, all the dysregulated lncRNAs were never reported in T2D. To uncover the potential function of these lncRNAs, we further investigated the relationship between differentially expressed lncRNAs and mRNAs constructing a gene co-expression network to identify correlated targets of dysregulated lncRNAs in our Han Chinese patients with T2D. Target mRNAs included LY86, S100A12, S100A8, CD36, TLR8, VNN2, and KCNJ15. We identified 10 pairs of co-expressed lncRNAmRNAs in our cohort. Previous studies reported that LY86 was involved in obesity, inflammation, and insulin resistance [31]. S100A8 and S100A12, belonging to the calgranulin gene family, play key immune response roles in inflammatory disorders, like diabetes and cardiovascular disease [32]. In addition, S100A8 can recruit macrophages to adipose tissue during inflammation and in the setting of hyperglycemia and obesity [33, 34]. It was reported that the elevated expression of TLR8 found in the adipose tissue of patients with obesity or T2D had consensus with inflammatory signatures and may represent an immune marker of metabolic inflammation [35]. Upregulation of CD36 is significantly associated with IR and hyperinsulinemia [36]. The vanin gene family member VNN2 encodes secreted and membrane-bound ectoenzymes that convert pantetheine into pantothenic acid and cysteamine [37]. Human VNN2 was characterized as a protein involved in the regulation of adherence and migration of neutrophils [38]. Whereas KCNJ15 is a type 2 diabetes-associated risk gene, overexpression of which suppresses insulin secretion in rat insulinoma (INS1) cells 


\section{Cellular Physiology Cell Physiol Biochem 2017;43:2367-2378

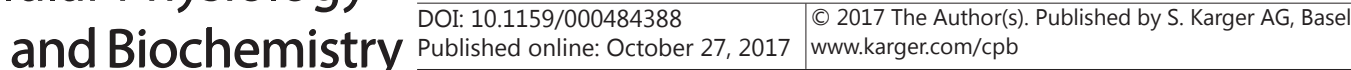 \\ Wang et al.: Long Non-Coding RNAs and Type 2 Diabetes in Han Chinese}

[39]. Although the roles of these target mRNAs in inflammation, IR, and insulin secretion are known, the functions of the co-expressed lncRNAs are not; however, we speculate they may have important roles in the pathophysiological processes of T2D by regulating expression of their co-expressed mRNA. Future studies are warranted to determine the precise regulatory mechanisms, which will provide a better understanding of the molecular processes involved in the pathogenesis of T2D.

Correlations between the top three upregulated lncRNAs and clinical features were identified in our cohort of patients. We found that these lncRNAs were correlated with metabolic indices, including FPG, 2hPG, HbA1c, and HOMA-IR. Fadista and colleagues [10] demonstrated an association between lncRNAs and HbA1c level, while those lncRNAs were specifically expressed in islet tissue. In the present study, we found that IncRNAs in peripheral whole blood were also associated with metabolic indices. Our findings suggest that these identified IncRNAs may play a role in the regulation of glycometabolism. One of the tested lncRNAs, n336109, was also negatively correlated with HOMA- $\beta$, a finding which indicates it may affect insulin secretion. Previous study reported that lncRNAs could affect apoptosis and insulin secretion in pancreatic $\beta$ cells [40]. Based on our CNC analysis, n336109 was positively co-expressed with KCNJ15, and additionally, microarray data showed that both n336109 and KCNJ15 were upregulated in our T2D group. Okamoto et al. [39]. reported that KCNJ15 overexpression decreases the insulin response to glucose in rat INS1 cells. The authors then demonstrated that KCNJ15 was a new susceptibility gene in Asian patients with T2D [41]. Although little is known about the function of n336109, its potential role in the pathogenesis of T2D is suggested not only by its expression change in our cohort of Han Chinese with T2D, but also by our correlation analysis using clinical data of our cohort.

Several limitations exist in this study. First, although the different expression patterns of lncRNAs suggest potential roles in T2D pathogenesis, the precise underlying regulatory mechanism(s) was not directly identified. Second, because these IncRNA expression profiles were not detected in other types of diabetes such as type 1 diabetes we cannot determine the specificity of these differentially expressed lncRNAs in T2D. Third, because IR is involved in adipose tissue, liver, and muscle, testing using samples of these tissues may be more suitable than peripheral blood. Nevertheless, to further elucidate the roles of IncRNAs in T2D, more laboratory and clinical research is needed.

\section{Conclusion}

This study characterizes the profiles of lncRNAs in a cohort of Han Chinese patients with T2D. Furthermore, although dysregulated lncRNAs may play potential roles in the pathogenesis of T2D through regulation of inflammation, IR, and insulin secretion, more research is needed to illuminate the functions of lncRNAs in the pathogenesis of T2D.

\section{Acknowledgements}

This work was supported by a grant from the National Natural Science Foundation of China (grant number 81560139).

\section{Disclosure Statement}

The authors have no conflicts of interest to declare.

\section{References}

1 International Diabetes Federation: IDF DIABETES ATLAS-7TH EDITION. http://www.diabetesatlas.org/ Accessed May 20, 2017.

-2 Mattick JS, Makunin IV: Non-coding RNA. Hum Mol Genet 2006;1:R17-29. 


\section{Cellular Physiology Cell Physiol Biochem 2017;43:2367-2378 and Biochemistry \begin{tabular}{c|c|c|} 
DOI: 101159/000484388 \\
Published online: October 27, 2017 & $\begin{array}{l}\text { O } 2017 \text { The Author(s). Published by S. Karger AG, Basel } \\
\text { www.karger.com/cpb }\end{array}$
\end{tabular}

3 Jia H, Osak M, Bogu GK, Stanton LW, Johnson R, Lipovich L: Genome-wide computational identification and manual annotation of human long noncoding RNA genes. RNA 2010;16:1478-1487.

4 Ponting CP, Oliver PL, Reik W: Evolution and functions of long noncoding RNAs. Cell 2009;136:629-641.

-5 Cesana M, Cacchiarelli D, Legnini I, Santini T, Sthandier O, Chinappi M, Tramontano A, Bozzoni I: A long noncoding RNA controls muscle differentiation by functioning as a competing endogenous RNA. Cell 2011;147:358-369.

6 Hu W, Alvarez-Dominguez Jr, Lodish HF, Lodish HF: Regulation of mammalian cell differentiation by long non-coding RNAs. EMBO Rep 2012; 13:971-983.

7 Gupta RA, Shah N, Wang KC, Kim J, Horlings HM, Wong DJ, Tsai M-C, Hung T, Argani P, Rinn JL, Wang Y, Brzoska P, Kong B, Li R, West RB, van de Vijver MJ, Sukumar S, Chang HY: Long non-coding RNA HOTAIR reprograms chromatin state to promote cancer metastasis. Nature 2010;464:1071-1076.

-8 Kumar V, Westra HJ, Karjalainen J, Zhernakova DV, Zhernakova Dv, Esko T Fau - Hrdlickova B, Almeida R, Zhernakova A, Reinmaa E, Vosa U, Hofker MH, Fehrmann RS, Fu J, Withoff S, Metspalu A, Franke L, Wijmenga C: Human disease-associated genetic variation impacts large intergenic non-coding RNA expression. PLos Genet 2013;9:e1003201.

-9 Ruan X: Long Non-Coding RNA Central of Glucose Homeostasis. J Cell Biochem 2016; 117:1061-1065.

10 Fadista J, Vikman P, Laakso EO, Mollet IG, Esguerra JL, Taneera J, Storm P, Osmark P, Ladenvall C, Prasad RB, Hansson KB, Finotello F, Uvebrant K, Ofori JK, Di Camillo B, Krus U, Cilio CM, Hansson O, Eliasson L, Rosengren AH, Renstrom E, Wollheim CB, Groop L: Global genomic and transcriptomic analysis of human pancreatic islets reveals novel genes influencing glucose metabolism. Proc Natl Acad Sci U S A 2014;111:13924-13929.

11 Qi M, Zhou Q, Zeng W, Shen M, Liu X, Luo C, Long J, Chen W, Zhang J, Yan S: Analysis of Long Non-Coding RNA expression of lymphatic endothelial cells in response to type 2 diabetes. Cell Physiol Biochem 2017;41:466-74.

12 Shi Z, Zhao C, Long W, Ding H, Shen R: Microarray expression profile analysis of long non-coding RNAs in umbilical cord plasma reveals their potential role in gestational diabetes-induced macrosomia. Cell Physiol Biochem 2015;36:542-54.

-13 Moran I, Akerman I, van de Bunt M, Xie R, Benazra M, Nammo T, Arnes L, Nakic N, Garcia-Hurtado J, Rodriguez-Segui S, Pasquali L, Sauty-Colace C, Beucher A, Scharfmann R, van Arensbergen J, Johnson PR, Berry A, Lee C, Harkins T, Gmyr V, Pattou F, Kerr-Conte J, Piemonti L, Berney T, Hanley N, Gloyn AL, Sussel L, Langman L, Brayman KL, Sander M, McCarthy MI, Ravassard P, Ferrer J: Human beta cell transcriptome analysis uncovers IncRNAs that are tissue-specific, dynamically regulated, and abnormally expressed in type 2 diabetes. Cell Metab 2012;16:435-448.

14 Gual P, Le Marchand-Brustel Y, Tanti JF: Positive and negative regulation of insulin signaling through IRS-1 phosphorylation. Biochimie 2005;87:99-109.

-15 Tanti JF, Ceppo F, Jager J, Berthou F: Implication of inflammatory signaling pathways in obesity-induced insulin resistance. Front Endocrinol (Lausanne) 2012;3:181.

-16 Palacios-Ortega S, Varela-Guruceaga M, Algarabel M, Ignacio Milagro F, Alfredo Martinez J, de Miguel C: Effect of TNF-Alpha on caveolin-1 expression and insulin signaling during adipocyte differentiation and in mature adipocytes. Cell Physiol Biochem 2015;36:1499-516.

17 Marques-Rocha JL, Samblas M, Milagro FI, Bressan J, Martinez JA, Marti A: Noncoding RNAs, cytokines, and inflammation-related diseases. Faseb J 2015;29:3595-3611.

-18 Zhou T, Meng X, Che H, Shen N, Xiao D, Song X, Liang M, Fu X, Ju J, Li Y, Xu C, Zhang Y, Wang L: Regulation of insulin resistance by multiple miRNAs via targeting the GLUT4 signalling pathway. Cell Physiol Biochem 2016;38:2063-2078.

19 Wang L, Zhang N, Pan HP, Wang Z, Cao ZY: MiR-499-5p contributes to hepatic insulin resistance by suppressing PTEN. Cell Physiol Biochem 2015;36:2357-2365.

20 Ma F, Li W, Tang R, Liu Z, Ouyang S, Cao D, Li Y, Wu J: Long non-coding RNA expression profiling in obesity mice with folic acid supplement. Cell Physiol Biochem 2017;42:416-26.

21 Alberti KG, Zimmet PZ: Definition, diagnosis and classification of diabetes mellitus and its complications. Part 1: diagnosis and classification of diabetes mellitus provisional report of a WHO consultation. Diabet Med 1998;15:539-553.

22 Chai V, Vassilakos A, Lee Y, Wright JA, Young AH: Optimization of the PAXgene blood RNA extraction system for gene expression analysis of clinical samples. J Clin Lab Anal 2005;19:182-188. 


\section{Cellular Physiology Cell Physiol Biochem 2017;43:2367-2378

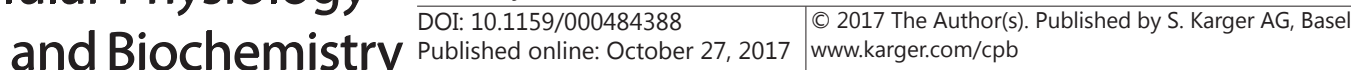 \\ Wang et al.: Long Non-Coding RNAs and Type 2 Diabetes in Han Chinese}

23 Cornelis MC, Hu FB: Gene-environment interactions in the development of type 2 diabetes: recent progress and continuing challenges. Annu Rev Nutr 2012;32:245-259.

-24 Gregor MF, Hotamisligil GS: Inflammatory mechanisms in obesity. Annu Rev Immunol 2011;29:415-445.

25 Lontchi-Yimagou E, Sobngwi E, Matsha TE, Kengne AP: Diabetes Mellitus and Inflammation. Curr Diab Rep 2013;13:435-444.

26 Magalhaes I, Kiaf B, Lehuen A: iNKT and MAIT Cell Alterations in Diabetes. Front Immunol 2015;6:341.

27 Lynch L: Adipose invariant natural killer T cells. Immunology 2014; 142:337-346.

28 Choi SS, Park J, Choi JH: Revisiting PPARgamma as a target for the treatment of metabolic disorders. BMB Rep 2014;47:599-608.

-29 Sarr O, Strohm RJ, MacDonald TL, Gaudio N, Reed JK, Foute-Nelong J, Dyck DJ, Mutch DM: Subcutaneous and Visceral Adipose Tissue Secretions from Extremely Obese Men and Women both Acutely Suppress Muscle Insulin Signaling. Int J Mol Sci 2017;18.

30 Mercer TR DM, Mattick JS: Long non-coding RNAs: insights into functions. Nat Rev Genet 2009;10:155-159.

-31 Su S, Zhu H, Xu X, Wang X, Dong Y, Kapuku G, Treiber F, Gutin B, Harshfield G, Snieder H, Wang X: DNA methylation of the LY86 gene is associated with obesity, insulin resistance, and inflammation. Twin Res Hum Genet 2014;17:183-191.

-32 Zhao P, Wu M, Yu H, Huang Y, Wang Y, Wang W, Yin W: Serum S100A12 levels are correlated with the presence and severity of coronary artery disease in patients with type 2 diabetes mellitus. J Investig Med 2013;61:861-866.

-33 Nagareddy PR, Murphy AJ, Stirzaker RA, Hu Y, Yu S, Miller RG, Ramkhelawon B, Distel E, Westerterp M, Huang LS, Schmidt AM, Orchard TJ, Fisher EA, Tall AR, Goldberg IJ: Hyperglycemia promotes myelopoiesis and impairs the resolution of atherosclerosis. Cell Metab 2013;17:695-708.

34 Sekimoto R, Fukuda S, Maeda N, Tsushima Y, Matsuda K, Mori T, Nakatsuji H, Nishizawa H, Kishida K, Kikuta J, Maijima Y, Funahashi T, Ishii M, Shimomura I: Visualized macrophage dynamics and significance of S100A8 in obese fat. Proc Natl Acad Sci U S A 2015;112:E2058-2066.

35 Ahmad R, Kochumon S, Thomas R, Atizado V, Sindhu S: Increased adipose tissue expression of TLR8 in obese individuals with or without type-2 diabetes: significance in metabolic inflammation. J Inflamm (Lond) 2016;13:38.

-36 Miquilena-Colina ME, Lima-Cabello E, Sanchez-Campos S, Garcia-Mediavilla MV, Fernandez-Bermejo M, Lozano-Rodriguez T, Vargas-Castrillon J, Buque X, Ochoa B, Aspichueta P, Gonzalez-Gallego J, GarciaMonzon C: Hepatic fatty acid translocase CD36 upregulation is associated with insulin resistance, hyperinsulinaemia and increased steatosis in non-alcoholic steatohepatitis and chronic hepatitis C. Gut 2011;60:1394-1402.

-37 Martin F, Malergue F, Pitari G, Philippe JM, Philips S, Chabret C, Granjeaud S, Mattei MG, Mungall AJ, Naquet P, Galland F: Vanin genes are clustered (human 6q22-24 and mouse 10A2B1) and encode isoforms of pantetheinase ectoenzymes. Immunogenetics 2001;53:296-306.

-38 Suzuki K, Watanabe T, Sakurai S, Ohtake K, Kinoshita T, Araki A, Fujita T, Takei H, Takeda Y, Sato Y, Yamashita T, Araki Y, Sendo F: A novel glycosylphosphatidyl inositol-anchored protein on human leukocytes: a possible role for regulation of neutrophil adherence and migration. J Immunol 1999;162:4277-4284.

39 Okamoto K, Iwasaki N, Nishimura C, Doi K, Noiri E, Nakamura S, Takizawa M, Ogata M, Fujimaki R, Grarup N, Pisinger C, Borch-Johnsen K, Lauritzen T, Sandbaek A, Hansen T, Yasuda K, Osawa H, Nanjo K, Kadowaki T, Kasuga M, Pedersen O, Fujita T, Kamatani N, Iwamoto Y, Tokunaga K: Identification of KCNJ15 as a susceptibility gene in Asian patients with type 2 diabetes mellitus. Am J Hum Genet 2010;86:54-64.

40 Yin DD, Zhang EB, You LH, Wang N, Wang LT, Jin FY, Zhu YN, Cao LH, Yuan QX, De W, Tang W: Downregulation of lncRNA TUG1 affects apoptosis and insulin secretion in mouse pancreatic beta cells. Cell Physiol Biochem 2015;35:1892-904.

41 Okamoto K, Iwasaki N, Doi K, Noiri E, Iwamoto Y, Uchigata Y, Fujita T, Tokunaga K: Inhibition of glucosestimulated insulin secretion by KCNJ15, a newly identified susceptibility gene for type 2 diabetes. Diabetes 2012;61:1734-1741. 